\title{
Effect of including a gas layer on the gel formation process during the drying of a polymer solution
}

\author{
Ramin Rabani ${ }^{1, *}$, Hatim Machrafi ${ }^{1}$, and Pierre Dauby ${ }^{1}$ \\ ${ }^{1}$ University de Liège, Thermodynamics of Irreversible Phenomena, Allée du 6-Août, 19, \\ BE-4000 Liège, Belgium \\ *Corresponding author: E-mail address:ramin.rabani@ulg.ac.be
}

\begin{abstract}
In this paper, we study the influence of the upper gas layer on the drying and gelation of a polymer solution. The gel is formed due to the evaporation of the binary solution into (inert) air. A one-dimensional model is proposed, where the evaporation flux is more realistically described than in previous studies. The approach is based on general thermodynamic principles. A composition-dependent diffusion coefficient is used in the liquid phase and the local equilibrium hypothesis is introduced at the interface to describe the evaporation process. The results show that high thickness of the gas layer reduces evaporation, thus leading to longer drying times. Our model is also compared with more phenomenological descriptions of evaporation, for which the mass flux through the interface is described by the introduction of a Peclet number. A global agreement is found for appropriate values of the Peclet numbers and our model can thus be considered as a tool allowing to link the value of the empirical Peclet number to the physics of the gas phase. Finally, in contrast with other models, our approach emphasizes the possibility of very fast gelation at the interface, which could prevent all Marangoni convection during the drying process.
\end{abstract}

Keywords: Drying of polymer solution, Gel formation, Gas layer, Thermodynamic evaporation flux

\section{Introduction}

The drying of liquid films of polymer solutions by evaporation of the solvent is an important process which is considered in numerous industrial applications including painting [1-3], coating [4-6], ink-jet printing [7-9], production of electronic-devices [10] and so on. The building of a basis for these technologies necessitates understanding the underlying physics of drying phenomena, and especially requires quantitative analysis of solvent evaporation in thin films. Therefore, a number of theoretical studies have been performed to develop models of these processes [11]. In the case of film drying, Bornside et al. [12] have taken into account the diffusion process in their model of spin coating and predicted numerically 
the formation of a solid "skin" at the free surface. De Gennes [13] analyzed the concentration profiles and the skin (crust) formation, and estimated theoretically the lifetime of the skin. Although his qualitative argument gives some important features of the skin, it is not possible to obtain a detailed information about the whole process of the skin formation. Tsige and Grest [14] undertook the molecular dynamics simulations of the evaporation process in polymer films. They reported the formation of a polymer density gradient at the film/vapor interface and also expressed that the rate of solvent evaporation from the film depends on the magnitude of this density gradient. However, they didn't discuss the restriction for the skin formation related to practical experiments. Reyes and Duda [15] proposed a Monte Carlo simulation for predicting the evolution of particle volume fraction during the drying process. The results indicated that under a slow evaporation rate the particles are able to crystallize, while for a faster evaporation a random packing is predicted. Routh and Zimmerman [16] studied the drying steps by considering a diffusion equation for the particles. The results demonstrated that strong diffusion causes a uniform film profile, while weak diffusion leads to skinning. Konig et al. [17] analyzed the effect of additional salt and displayed that the higher salt concentrations result in high likelihood of the skin formation. A possible explanation was provided by Sarkar and Tirumkudulu [18] who demonstrated how the charge on colloidal particles increases the particle diffusivity and thus changes the volume fraction profile during drying. Another way to change the diffusion coefficient of colloidal particles is with free polymer (soluble polymer) [19]. It was shown how adding polymer to a silica water solution lowers the diffusion coefficient and effectively increases the Peclet number, enhancing non-uniform drying. Ozawa et al. [20] modeled a diffusion-type equation with regard to the gelation effect of the solution in polymer solution undergoing evaporation of the solvent at the free surface. The results implied that the drying dynamics of polymer solutions is strongly related to the gelation, which leads to a great enhancement in the diffusion coefficient of polymers. Hennessy et al. [21] presented a twophase model for volatile solvent and a nonvolatile polymer in a thin-film. Accordingly, they formulated a two-phase model to describe an evaporating solvent-polymer mixture and then employed it to investigate the interplay between gravity, evaporation, and skin formation. They found that the shortest drying time occurs in the limit of strong gravitational effects due to the rapid formation of a bilayer with a polymer-rich lower layer and a solvent-rich upper layer, while drag leads to the formation of a polymer-rich skin below the free surface and causes the drying time to increase significantly. In another study, Hennessy et al. [22] considered a model of solvent evaporation in a thin film comprised of volatile solvent and a nonvolatile solute which can be used to predict the dynamics of drying and film formation.

Undeniably, the numerous models proposed in previous studies to study the drying process have provided the opportunity to achieve a wealthy insight in this problem. However, the validity of some simplifying assumptions can still be questioned. For instance, many studies 
use a phenomenological law to describe the evaporation process, which amounts to introducing a Peclet number to quantify the importance of evaporation. However, the value of that number that can truly capture the drying process number for a given practical situation is not easy to determine. Another important question that has not been examined in the previous studies mentioned above is the possibility of a very fast gelation at the liquidgas interface, which would prevent all Marangoni convection during the drying of the film.

To address these questions, the present study proposes a more realistic model of the evaporation flux, which is based on thermodynamic principles, as well as on the physics of the interaction between the gas phase and the liquid phase. A similar approach was already proposed in [23] for a binary liquid but here we consider the case of a polymer solution in order to include gelation phenomena. A composition dependent diffusion coefficient is introduced, which allows to describe gelation and the formation of a skin phase. In order to assess the importance of using a more realistic description of the evaporation flux, the present model is compared with other ones including linear and nonlinear models. In addition, the effect of various thickness of gas layer has been examined on the evaporation process.

\section{Formulation of the problem}

The studied system, shown in Fig. 1, is comprised of a mixture of a volatile solvent and a non-volatile polymer placed on a flat solid and non-permeable substrate, under a layer of inert air. Our model of the evaporation process introduces a thermodynamics-based expression for the evaporation flux and this approach will be compared with two models from the literature that express the evaporation flux, respectively, as a linear [20] and a nonlinear [22] function of the mass fraction at the interface. In Fig. 1 and in the equations presented below, $z$ is the vertical coordinate, whereas $h$ is the total height of the solution and the possible skin, $h_{g}$ is the thickness of the liquid part, while $H$ is the total height of the liquid-skin-gas system. The gel thickness is thus given by $h-h_{g}$. The surface tension at the liquid-gas interface is assumed to be sufficiently strong for deformations of the film surface to be negligible. The time dependent thickness of the film can be depicted by a function of time $h(t)$. Diffusion is assumed to be the only mechanism of mass transport within the bulk and a Fick law is used, with a composition dependent diffusion coefficient. Any temperature variations, such as those resulting from evaporative cooling, are presumed to be sufficiently small so that the system can be treated as isothermal [22,24]. We will also consider that the solvent and polymer densities are not too different, which allows to consider that during the drying process, the mixture keeps a constant density, equal to the initial density of the liquid mixture. Similarly, the density in the gas phase will also be assumed to be independent of the solvent concentration. We will also neglect the Stefan flow in the gas, but not at the 
liquid-gas interface. This is a reasonable assumption in case the solvent vapor content is low [25], which can safely be assumed when the solvent considered is water, whose saturation pressure is low with respect to the gas pressure. In situations for which the concentration of the vapor in the gas is high due to a high vapor pressure (e.g. HFE -7100 [25]), the Stefan flow should of course be added in the description Finally, we will not describe hydrodynamic instabilities and convection is thus not taken into account. In this context, and because the system is horizontally uniform, a one-dimensional description is proposed.

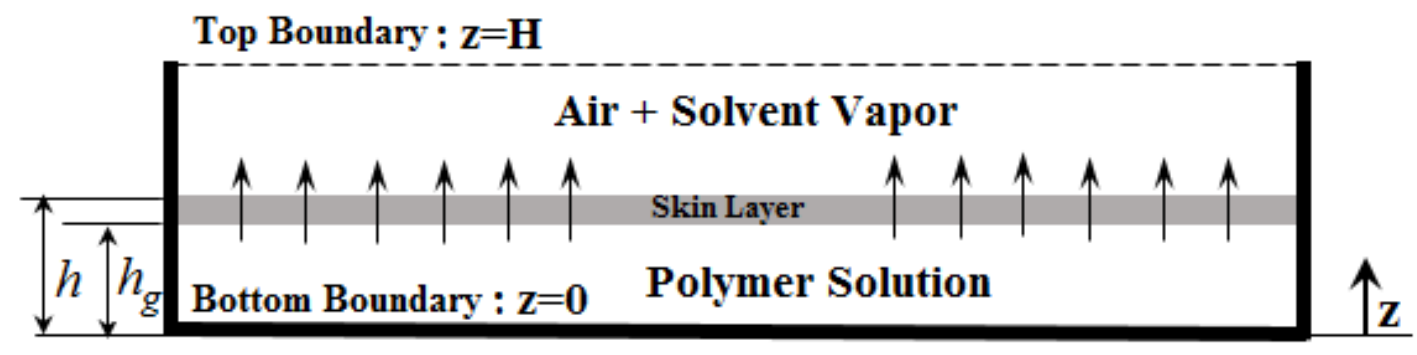

Fig. 1. Schematic of studied system

\subsection{Thermodynamic model}

A thermodynamic model of the evaporation process is built by considering the exchange of solvent with the gas phase. The evaporation process is described by a mass flux $J$ that must balance the diffusive fluxes at the film surface. The total system with size $H$ consists of two phases. The first phase is the gas layer, $h \leq z \leq H$, where the diffusion of solvent vapor takes place through the air and the gas mass fraction (of the solvent) $\mathrm{Cg}$ follows the diffusion equation

$$
\frac{\partial C_{g}}{\partial t}=D_{g}\left(\frac{\partial^{2} C_{g}}{\partial z^{2}}\right)
$$

where $D_{g}$ is the assumed constant diffusion coefficient in gas phase. The second part of the system is the liquid and gel layer, $0 \leq z \leq h$, where the conservation equation describing the solvent mass concentration $\left(C_{l}\right)$ with variable diffusion coefficient in the drying process obeys

$$
\frac{\partial C_{l}}{\partial t}=\frac{\partial}{\partial z}\left(D_{l} \frac{\partial C_{l}}{\partial z}\right)
$$

where the composition dependent diffusion coefficient $D_{l}$ for the polymer solution is given by [20]: 


$$
D_{l}= \begin{cases}D_{\circ} C_{l}\left(1-C_{l}\right)^{p} & C_{l}>C_{\text {gel }} \\ D_{\circ}\left[C_{l}\left(1-C_{l}\right)^{p}+d C_{l}\left(1-C_{l}\right)^{p}\left(C_{\text {gel }}-C_{l}\right)^{b}\right] & C_{l} \leq C_{\text {gel }}\end{cases}
$$

where $D_{o}$ is the diffusion coefficient of pure solvent, while $C_{g e l}$ is the solvent mass fraction below which gelation takes place (here we will choose $C_{g e l}=0.5$ ). As in [20], we take $b=p=3$ and we also take $d=1000$ in order to have a clear gelation transition. It is important to stress here that a clear definition of a gel, and the corresponding modelling of this material, is a delicate subject, which remains somewhat controversial [26]. However, such interesting questions fall outside the scope of our work and together with [20], we will simply admit here that what we call a gel is described by Eq. 3. Note also that in Eqs. (1) and (2), we have used Fick's law. For the liquid phase, which is a polymer-solvent mixture, this law is not quantitatively precise for high polymer concentrations, and more complex descriptions of the thermodynamics of the non-ideal mixtures should be considered [27]. However, for the purpose of this paper, the qualitative description based on Fick's law is largely sufficient.

The boundary conditions for solving Eqs. (1) and (2) are the following. A no-flux boundary condition is applied at the non-permeable substrate $(z=0)$ :

$$
\frac{\partial C_{l}}{\partial z}=0
$$

A fixed constant value of the solvent mass fraction is imposed at the top boundary of the gas layer and, except otherwise stated, we will consider a zero humidity for the air far from the interface (i.e. $z=H$ ). One thus has:

$$
C_{g}=0
$$

The boundary conditions at the liquid-gas interface can be deduced from the principle of mass conservation. First, we assume that the polymer does not evaporate. Its flux across the moving boundary is thus zero and one has:

$$
-\left.\rho_{l} D_{l} \frac{\partial\left(1-C_{l}\right)}{\partial z}\right|_{z=h}-\rho_{l}\left(1-C_{l}\right) \frac{d h}{d t}=0
$$

where $\rho_{l}$ is the (assumed constant) liquid density. The conservation of solvent then imposes

$$
-\left.\rho_{l} D_{l} \frac{\partial C_{l}}{\partial z}\right|_{z=h}-\rho_{l} C_{l} \frac{d h}{d t}=J
$$

Combining the previous two equations directly provides the following alternative expressions of the above boundary conditions at $z=h(t)$ :

$$
\left(\frac{d h}{d t}\right)=-\frac{J}{\rho_{l}}
$$




$$
-\left.\rho_{l} D_{l} \frac{\partial C_{l}}{\partial z}\right|_{z=h}=J\left(1-C_{l}\right)_{z=h}
$$

Considering that air cannot be absorbed in the liquid and using a procedure similar to that used in the liquid, one can obtain the following additional boundary condition at $z=h(t)$ :

$$
-\left.\rho_{g} D_{g} \frac{\partial C_{g}}{\partial z}\right|_{z=h}=J\left(1-C_{g}\right)_{z=h}
$$

where $\rho_{g}$ is the gas density.

To describe local equilibrium at the liquid-gas interface, we will use Raoult's law [28]. This law is normally valid for ideal solutions and it is not sure that it is strictly correct in our case, especially when the concentration of the polymer becomes high. However, in our approach which is mainly qualitative and interested in general physical mechanisms, we will consider this law as a sufficient approximation. Since only the solvent can cross the interface, Raoult's law takes the form:

$$
y_{g} p_{g}=y_{l} p_{\text {sat }}
$$

where $y_{g}$ and $y_{l}$ are the molar fractions of the solvent in the gas and liquid phases, respectively, $p_{g}$ is the total pressure of gas at the interface, and $p_{\text {sat }}$ is the saturation pressure of the pure solvent (at the temperature of the experiment). In terms of mass fraction, Raoult's law can be rewritten as follows:

$$
\frac{C_{g} \delta_{a s}}{1+C_{g}\left(\delta_{a s}-1\right)}=\frac{C_{l} \delta_{p a}}{1+C_{l}\left(\delta_{p a}-1\right)} \frac{p_{s a t}}{p_{g}}
$$

where $\delta_{p a}=\frac{M_{p}}{M_{s}}$ is the polymer to solvent molecular mass ratio, while $\delta_{a s}=\frac{M_{a}}{M_{s}}$ is the air to solvent molecular mass ratio.

\subsection{Non-dimensionalization of the equations}

It is convenient to rewrite the equations in a non-dimensional form. The initial film thickness of the liquid, $h_{o}$, is taken as the characteristic length and while the diffusion time scale in pure solvent, $\tau_{l}=h_{o}^{2} / D_{o}$, is chosen as time scale. In these new units, the equations take the form

$$
\begin{aligned}
& \frac{\partial C_{l}}{\partial t}=\frac{\partial}{\partial z}\left(\tilde{D}_{l} \frac{\partial C_{l}}{\partial z}\right) \\
& \frac{\partial C_{g}}{\partial t}=D \frac{\partial^{2} C_{g}}{\partial z^{2}}
\end{aligned}
$$




$$
\begin{aligned}
\tilde{D}_{l} & = \begin{cases}C_{l}\left(1-C_{l}\right)^{p} & C_{l}>C_{\text {gel }} \\
C_{l}\left(1-C_{l}\right)^{p}+d C_{l}\left(1-C_{l}\right)^{p}\left(C_{g e l}-C_{l}\right)^{b} & C_{l} \leq C_{\text {gel }}\end{cases} \\
\frac{\partial C_{l}}{\partial z}=0, z=0 \quad \& \quad C_{g}=0, z=H &
\end{aligned}
$$

where $z$ and $H$ are now non-dimensional, with $D=D g / D_{0}$. Note that the non-dimensional thickness $h$ is now such that $h(0)=1$. The boundary conditions take the form:

$$
\begin{aligned}
& -\left.\tilde{D}_{l} \frac{\partial C_{l}}{\partial z}\right|_{z=h}=\zeta\left(1-C_{l}\right)_{z=h} \\
& -\left.\frac{D}{\rho} \frac{\partial C_{g}}{\partial z}\right|_{z=h}=\zeta\left(1-C_{g}\right)_{z=h}
\end{aligned}
$$

$$
\left(\frac{d h}{d t}\right)=-\zeta
$$

where $\rho=\frac{\rho_{l}}{\rho_{g}}$, while the dimensionless mass flux $\zeta$ is given by $\zeta=\frac{J h_{\circ}}{\rho_{l} D_{\circ}}$. Raoult's law is already given by (12):

$$
\frac{C_{g} \delta_{a s}}{1+C_{g}\left(\delta_{a s}-1\right)}=\frac{C_{l} \delta_{p a}}{1+C_{l}\left(\delta_{p a}-1\right)} \frac{p_{s a t}}{p_{g}}
$$

\subsection{Linear and nonlinear models}

Let us now briefly describe two other phenomenological models of evaporation to which ours will be compared [20, 22]. For these models, the behavior of the gas layer is no longer described and a simple phenomenological law is proposed to describe the evaporation flux. In both cases, the flux depends on the concentration of the evaporating component in the liquid along the interface. For solvent-polymer mixtures, linear and non-linear expressions were proposed by Ozawa et al. [20] and Hennessy et al. [22] respectively. The corresponding non-dimensional evaporation fluxes are denoted $\zeta_{L}$ and $\zeta_{n L}$ and take the form:

$$
\begin{aligned}
& \zeta_{L}=\left.P e C_{l}\right|_{z=h} \\
& \zeta_{n L}=P e\left[C_{l} e^{1-C_{l}+\chi\left(1-C_{l}\right)^{2}}\right]_{Z=h}
\end{aligned}
$$

where $P e$ is the so-called Peclet number and $\chi$ is the interaction parameter. This nondimensional number is the ratio of the diffusive time scale $\tau_{\text {diff }}=\frac{h_{0}^{2}}{D_{0}}$ to the mass transfer time scale $\tau_{m t}=\frac{\rho_{l} h_{0}}{J_{e v}}$ where $J_{e v}$ is a phenomenological constant. One thus has $P_{e}=\frac{J_{e v} h_{0}}{\rho_{l} D_{0}}$. 


\section{Results and discussion}

The models presented above were solved using an explicit numerical method and applied to a binary mixture of PVA (polyvinyl-alcohol)-water system. The density of PVA (1270 $\mathrm{kg} / \mathrm{m}^{3}$ ) is a bit different from that of water but the effect of this difference will not be taken into account, as explained in Section 2. In the gas phase, the binary diffusion coefficient is fixed to $2.54 \times 10^{-5} \mathrm{~m}^{2} / \mathrm{s}$ [29]. Moreover, the total pressure of the gas is equal to the atmospheric pressure and the saturation pressure of water at a constant temperature of $300^{\circ} \mathrm{K}$ is $p_{\text {sat }}=3.52 \times 10^{-2}$ atm [29].

Before evaporation starts, it is assumed that the liquid film is well mixed, corresponding to spatially uniform distribution of solvent and solute in the liquid and the corresponding solvent concentration is $C_{l i}$. Similarly, in the gas phase the solvent concentration is also assumed uniformed and equal to $C_{g i}$. Then suddenly at $t=0$, the two phases are brought into contact and evaporation starts with the following initial conditions:

$C_{l}(z, 0)=C_{l i} \quad, \quad 0 \leq z \leq h$

$C_{g}(z, 0)=C_{g i}, \quad h \leq z \leq H$

$h(0)=1$

Table 1. Physical properties and parameters values of the problems

\begin{tabular}{|c|c|c|c|}
\hline Physical property & Value & parameter & Value \\
\hline$\rho_{\text {water }}$ & $1000 \mathrm{~kg} / \mathrm{m}^{3}$ & $C_{l i}$ & $0.7,0.51$ \\
\hline$\rho_{\text {PVA }}$ & $1270 \mathrm{~kg} / \mathrm{m}^{3}$ & $C_{g i}$ & 0 \\
\hline$D_{g}$ & $2.54 \times 10^{-5} \mathrm{~m}^{2} / \mathrm{s}$ & $C_{g e l}$ & 0.5 \\
\hline$p_{\text {sat }}$ & $3.52 \times 10^{-2} \mathrm{~atm}$ & $P e$ & $1<P e<20$ \\
\hline$p_{g}$ & $1 \mathrm{~atm}$ & $H$ & $2,5,9,21,101$ \\
\hline
\end{tabular}

In the following, we present the general results of our study for which the physical properties of the fluids and the parameters corresponding to the simulations are summarized in Table 1. First, the time evolution of the system as predicted by our model is presented. Then these results are compared with those corresponding to the linear and nonlinear models.

\subsection{General results}

A typical numerical solution of Eqs. (13) and (14) is represented in Fig. 2. The uniform initial mass fractions of liquid and gas layers were set to $C_{l i}=0.7$ and $C_{g i}=0$, respectively. The total thickness of the two-layer system was considered to be $H=2$. Fig. 2 a and b show the time evolution of the profile $C_{l}$ and the contour plot of the binary liquid layer thickness in the drying process and gel formation, respectively. At the very beginning (small $t$ ), a 
polymer-rich region is formed near the free surface. Soon after, due to the high evaporation rate, $C_{l}(z, t)$ reaches $C_{g e l}$ and a gel layer in which $C_{l}(z, t) \leq C_{g e l}$ is formed. As drying proceeds, the gel layer develops and get thicker, before the entire lower layer finally tends towards gelation. At this moment, the evaporation rate considerably reduces, until only the polymer is present and evaporation ceases.
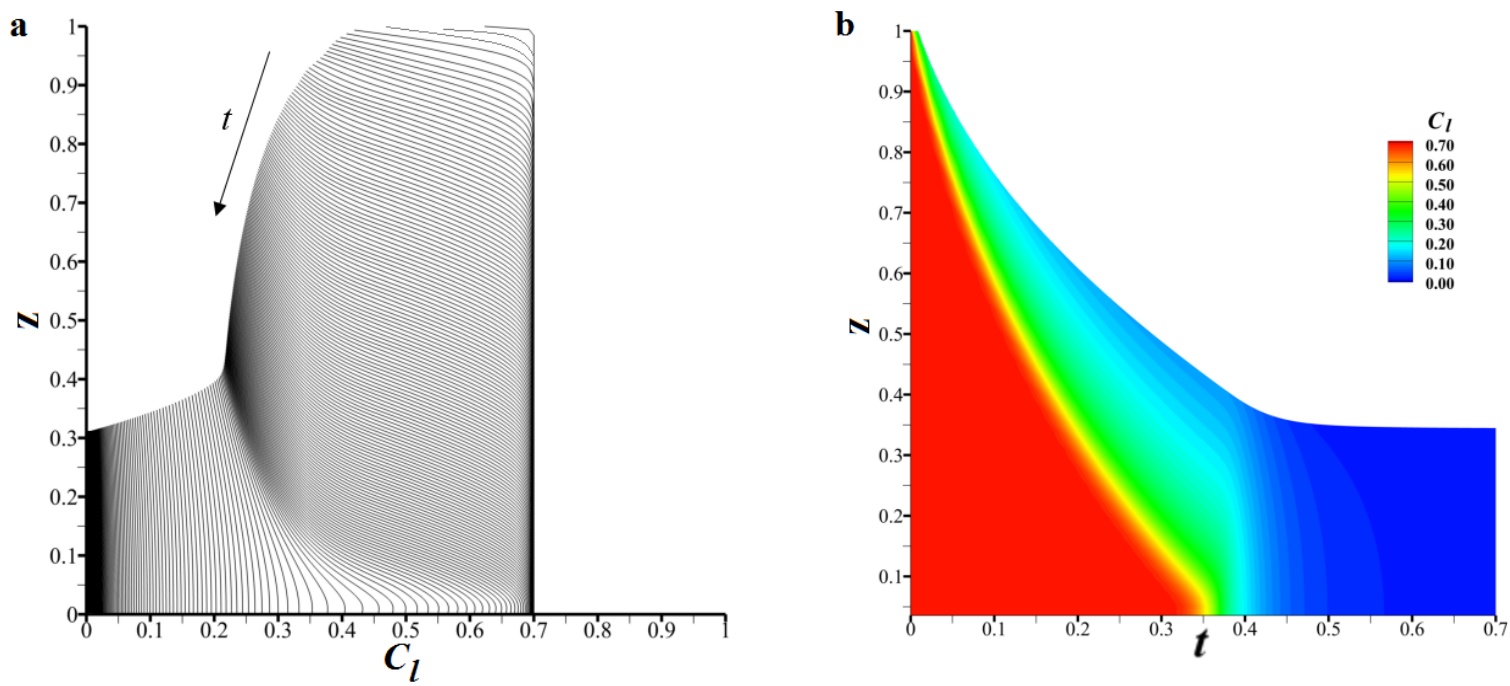

Fig.2 (a) Time evolution of the solvent mass fraction profile $C_{l}(z, t)$ and (b) contour plot of the solvent mass fraction $C_{l}(z, t)$

Fig. 3 shows the time evolution of the binary liquid layer thickness $h$ for different values of $H$, which allows to emphasize the effect of the gas thickness on the evaporation process. As expected, thick gas layer corresponds to reduced evaporation rates, which leads to a longer drying time.

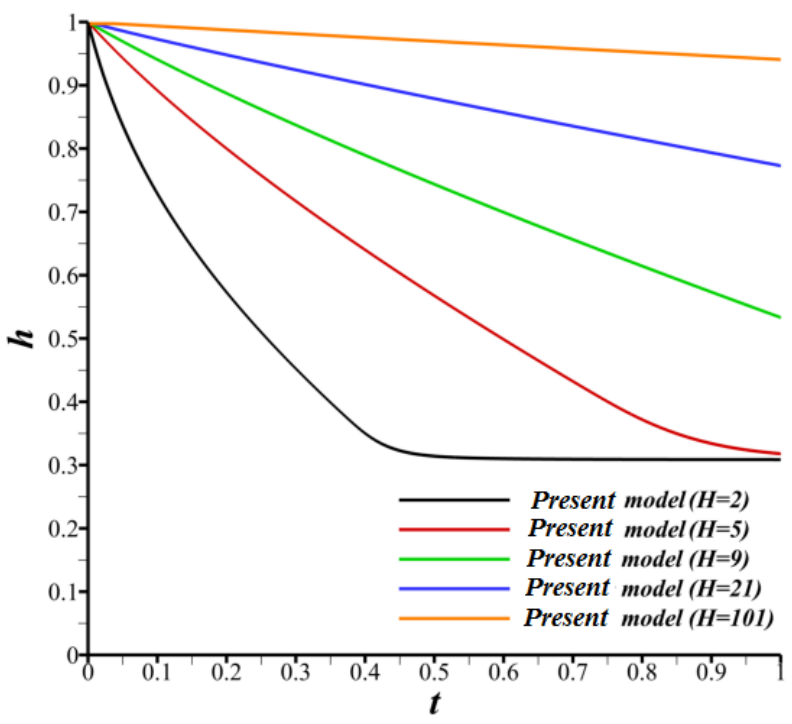

Fig. 3. Effect of the gas layer thickness on the time evolution of the mixture height $h$. 


\subsection{Immediate gelation}

When the binary liquid and gas phase, which are not in equilibrium with respect to one another, are suddenly brought into contact at $t=0$, a very strong evaporation is expected in the very beginning. The resulting important decrease of the liquid solvent concentration at the interface could thus result in an immediate gelation. Of course, the detailed physics of the first instants after the discontinuity is difficult to describe precisely and a simplifying assumption must be introduced to study the system [29]. In order to examine the possibility of a direct gelation of the interface, we will assume that right after the two systems are brought into contact, an equilibrium between the liquid and gas phases is reached at $t=0^{+}$, which means that a jump of the two interfacial concentrations occurs in order to satisfy the boundary conditions. This initial jump can be considered as a disturbance for the two phases, and the corresponding perturbations in the two phases will then propagate away from the interface and create two boundary layers. In the very beginning, the perturbations remain close to the interface, and the two phases can thus be considered as infinitely deep. We will now show that a self-similar model of the system can be developed, which will allow determining the values of the interfacial concentrations after the jump. As a first step to build the self-similar model, we consider a vertical axis that follows the moving interface. This amounts to considering the following change of variables: $\tau=t$ and $\xi=z-h(t)$, where $h(t)$ is the position (in the original $z$-coordinate) of the moving interface. Eqs. (13) and (14) then take the form:

$$
\begin{aligned}
& \frac{\partial C_{l}}{\partial \tau}-\frac{\partial C_{l}}{\partial \xi} \frac{d h}{d \tau}=\frac{\partial}{\partial \xi}\left(\tilde{D}_{l} \frac{\partial C_{l}}{\partial \xi}\right) \\
& \frac{\partial C_{g}}{\partial \tau}-\frac{\partial C_{g}}{\partial \xi} \frac{d h}{d \tau}=D \frac{\partial^{2} C_{g}}{\partial \xi^{2}}
\end{aligned}
$$

Then a similarity parameter $\eta \in[0,+\infty]$ can be introduced, with $\eta=-\xi / \sqrt{\tau}$ in the liquid and with $\eta=\xi / \sqrt{\tau}$ in the gas. In terms of this parameter, one can easily check that the equations and boundary conditions become:

$$
\begin{aligned}
& \tilde{D}_{l} \frac{\partial^{2} C_{l}}{\partial \eta^{2}}+0.5 \eta \frac{\partial C_{l}}{\partial \eta}+\frac{\partial \tilde{D}_{l}}{\partial C_{l}}\left(\frac{\partial C_{l}}{\partial \eta}\right)^{2}+\frac{\partial C_{l}}{\partial \eta} \frac{\left(\tilde{D}_{l} \frac{\partial C_{l}}{\partial \eta}\right)_{\eta=0}}{1-\left.C_{l}\right|_{\eta=0}}=0 \\
& \frac{\partial^{2} C_{g}}{\partial \eta^{2}}+\frac{0.5}{D} \eta \frac{\partial C_{g}}{\partial \eta}+\frac{\partial C_{g}}{\partial \eta} \frac{\left(\frac{D}{\rho} \frac{\partial C_{g}}{\partial \eta}\right)_{\eta=0}}{1-\left.C_{g}\right|_{\eta=0}}=0
\end{aligned}
$$


$\left.\frac{D}{\rho\left(1-C_{g}\right)_{z=0}} \frac{\partial C_{g}}{\partial \eta}\right|_{\eta=0}=-\left.\frac{\tilde{D}_{l}}{\left(1-C_{l}\right)_{z=0}} \frac{\partial C_{l}}{\partial \eta}\right|_{\eta=0}$

$\left[\frac{C_{g} \delta_{a s}}{1+C_{g}\left(\delta_{a s}-1\right)}\right]_{\eta=0}=\left[\frac{C_{l} \delta_{p a}}{1+C_{l}\left(\delta_{p a}-1\right)} \frac{p_{s a t}}{p_{g}}\right]_{\eta=0}$

$\left.C_{l}\right|_{\eta=\infty}=C_{l i}$

$\left.C_{g}\right|_{\eta=\infty}=C_{g i}$

This system of equations is a boundary value problem, but it is worth emphasizing that the values of the unknown fields and of their derivatives at the interface also appear in the differential equations. Defining unknown parameters equal to these quantities, the problem can be directly solved using the MATLAB bvp4c function. For evident numerical reasons, the boundary condition at infinity were expressed far from the interface, but at a finite distance which was chosen as $\eta=10$.

The results of our analysis are summarized in Fig. 4, for which ambient pressure and temperature were assumed $\left(T_{a m b}=300^{\circ} \mathrm{K}, P_{a m b}=1 \mathrm{~atm}\right)$. The initial concentration in the liquid is fixed to $C l_{\mathrm{i}}=0.51$. We have then considered several initial concentrations in the gas phase, which were equivalently expressed in terms of a relative humidity. To define precisely this quantity, we need to introduce first the saturated gas concentration $\left(C_{g s}\right)$ at ambient conditions.

$$
\frac{C_{g s} \delta_{a s}}{1+C_{g s}\left(\delta_{a s}-1\right)}=\frac{C_{l i} \delta_{p a}}{1+C_{l i}\left(\delta_{p a}-1\right)} \frac{p_{s a t}}{p_{g}}
$$

Then the initial relative humidity $R H \%$ of the gas is defined in terms of the initial concentration $C_{g i}$ by $R H \%=100 \% \times C_{g i} / C_{g s}$. In Fig. 4, we have plotted the liquid interfacial solvent concentration $\mathrm{Cl}_{\Sigma}$ at $t=O^{+}$, i.e. the liquid interfacial concentration after the jump, for several initial relative humidities in the gas phase. The results show that for low initial humidity in the gas, immediate gelation can take place at the interface, as soon as the two liquid and gas layers are brought into contact. This immediate gelation is of course a consequence of the very strong evaporation that takes place in the system when the initial solvent concentration in the gas is very low. In these circumstances, the initial jump thus prevents all Marangoni instabilities and convection. Finally let us mention that we have also checked that neglecting the motion of the liquid-gas interface, i.e. setting the term $d h / d \tau \equiv$ 0 in Eqs. (24) and (25), changes the results of Fig. 4 by less than $0.1 \%$ (in fact, the results with $d h / d \tau=0$ were used as initial guess for the numerical procedure in bvp4c when $d h / d \tau \neq 0)$. 


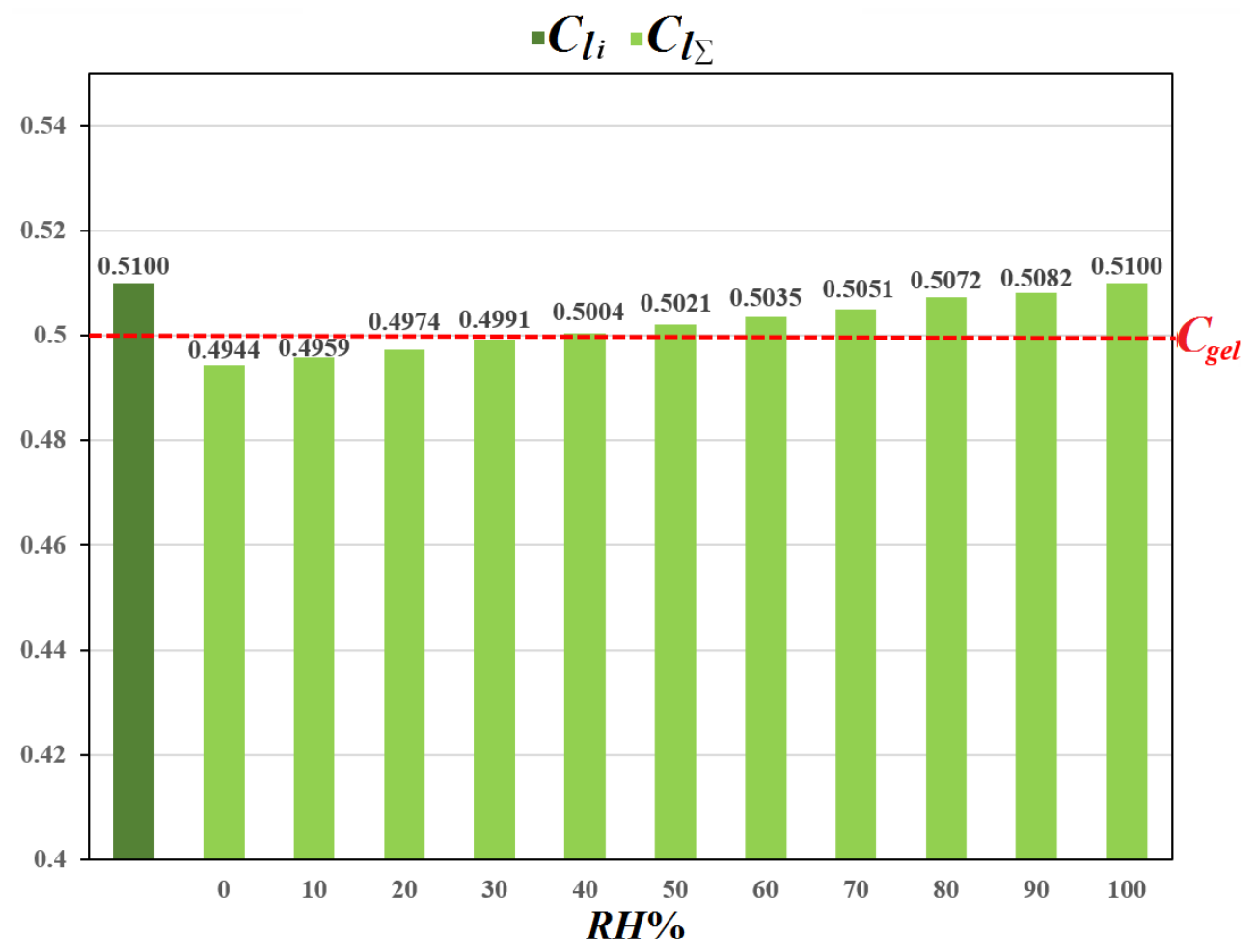

Fig. 4. Interfacial liquid mass fraction $\left(C l_{\Sigma}\right)$ after the jump for various initial humilities $(R H \%)$ in the gas phase (ambient pressure and temperature in the gas). The first bar (dark green) gives the value of the mass fraction in the liquid before the contact

\subsection{Comparison with other models}

To compare our model with the phenomenological models described before, we have analyzed the time evolution of the thickness $h(t)$ as predicted in the different approaches. In Figs. 5 (a) and (b), we have plotted $h(t)$ respectively for the linear and nonlinear models and we have also considered several values of $P e$ and two values of $H$. In all figures, the results of our model are plotted using black full lines. Of course, we note that the final value of $h$, corresponding to $t \rightarrow \infty$, is independent of the model and also of $H$ and of $P e$. We also observe that increasing the Peclet number gives rise to a faster decrease of the thickness and that for a given $P e$, evaporation is faster for the linear model that for the nonlinear one. Eventually, it is important to mention that is always possible to determine a value of the Peclet number such that the evolutions predicted by our model and that coming from the linear or nonlinear model are quite similar (for the linear and nonlinear models, these values are respectively $P e=4$ and $P e=11$ for $H=2$ and at $P e=1.2$ and $P e=3$ for $H=5$ ). This is an important fact since it allows to relate the value of the purely empirical Peclet number to the true physical quantities that are considered in our approach (diffusion in the gas, condition at the top of the gas, etc.). However even if the general trends predicted by our model and by the phenomenological ones are the same for appropriate $P e$, it is worth emphasizing important differences, especially in the very beginning of the drying process. Indeed, the phenomenological models do not allow jump to occur at the very beginning, because the 
evaporation flux, which is determined by the interfacial solvent concentration, remains always finite in these models, while it is theoretically infinite at $t=0$ in our approach. As an illustration of this difference, Fig. 6 is a plot of the time evolution of the interfacial solvent concentration in the liquid for our model and also for the linear model with $P e=14$. We have considered a zero initial humidity in the gas and an initial concentration in the liquid equal to $C l_{\mathrm{i}}=0.51$. After the jump, the interfacial concentration becomes 0.4944 in our model (see Fig. 4). Fig. 6 then clearly emphasizes that even if the long-time behavior predicted by the two models are quite similar, the evolutions for small times are clearly different. In particular, immediate gelation takes places for our model, while this phenomenon is always impossible for the phenomenological models. Note also that the sudden slope change around $t=0.29$ is due to the fact that the bottom of the gel layer reaches the substrate $(z=0)$ at that time.
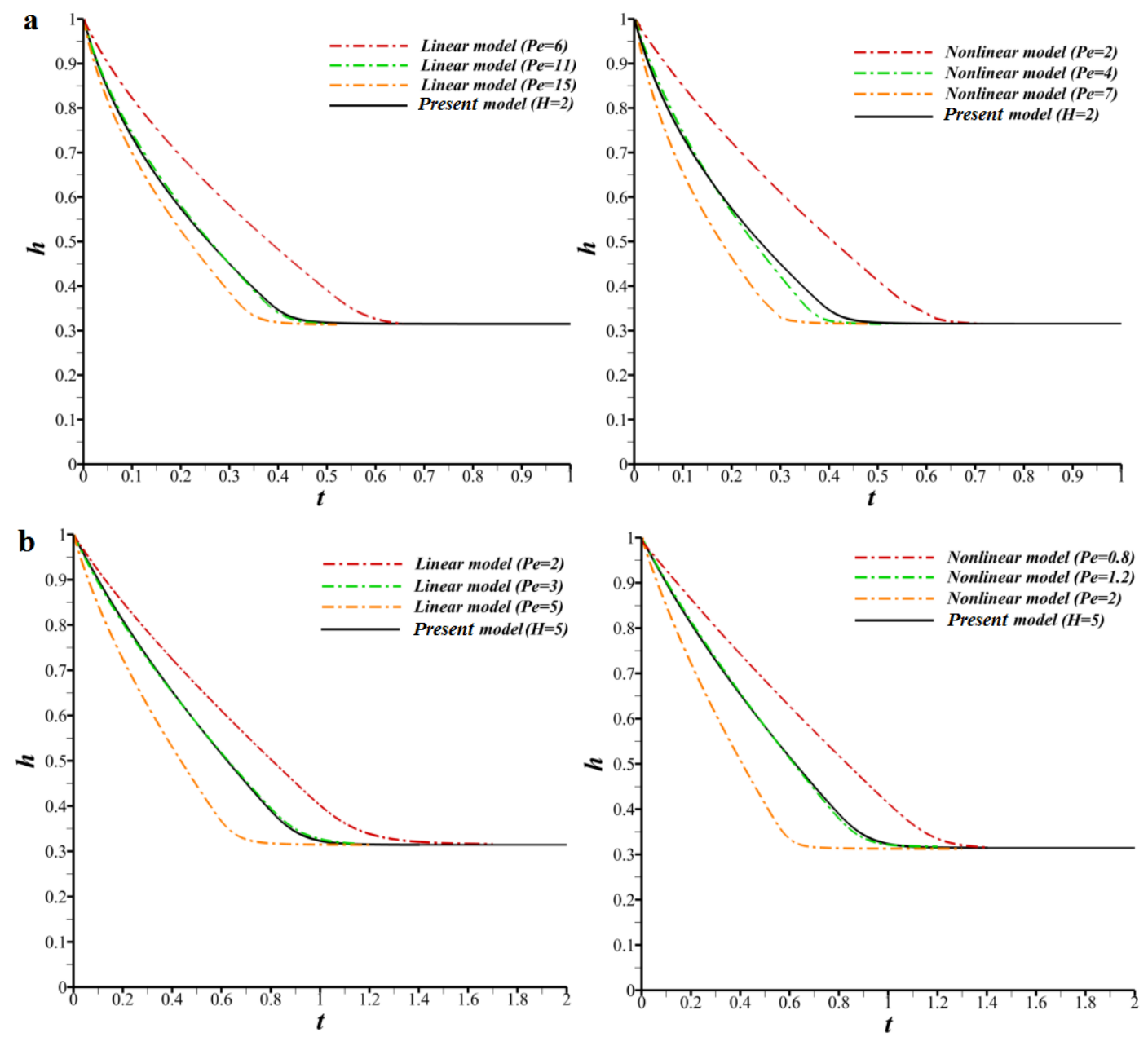

Fig. 5. Time evolution of the thickness: comparison between our model and the linear and nonlinear models (left and right panels respectively) for different Peclet numbers; (a) $H=2$ and (b) $H=5$. 


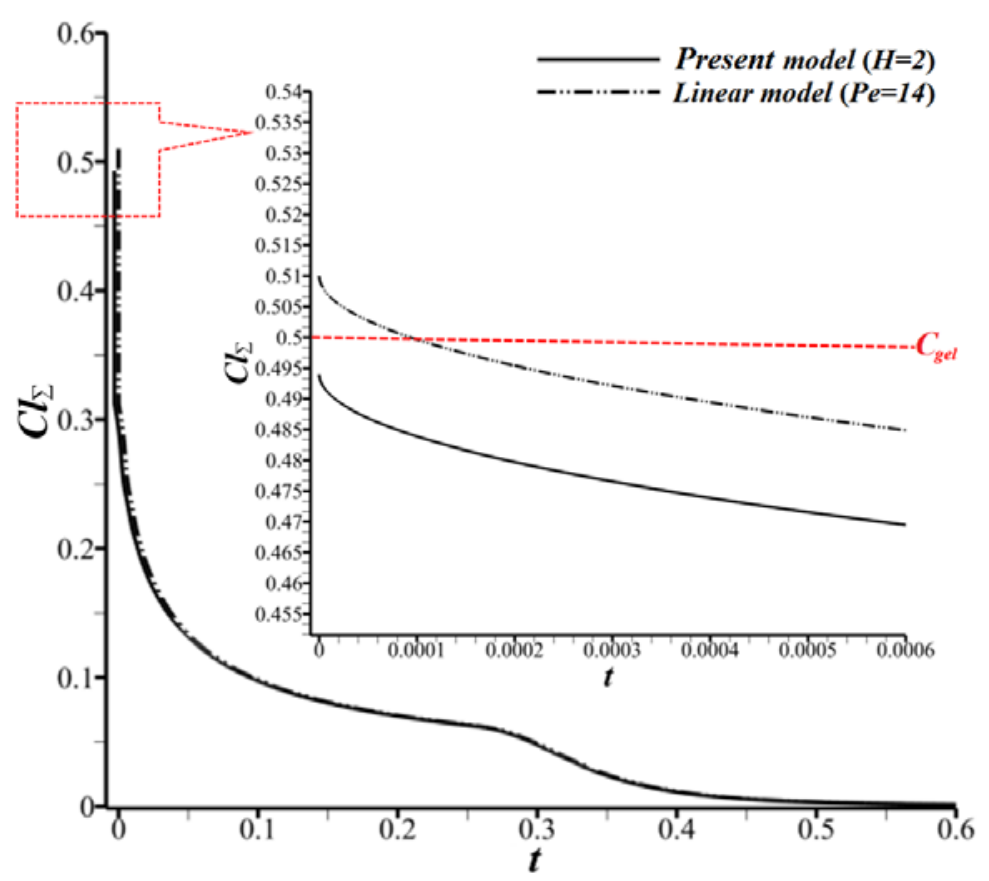

Fig. 6. Liquid mass fraction $\left(\mathrm{Cl}_{\Sigma}\right)$ at interface as a function of time for the linear (with $\left.\mathrm{Pe}=14\right)$ and present models $(R H \%=0$ and $C l i=0.51)$.

\section{Other initial conditions}

From an experimental point of view, bringing suddenly the 2 phases into contact at $t=0$ is not quite realistic, rendering comparisons with experiments virtually impossible. For this reason, we have also examined the possibility of different initial conditions and a different start of the drying process, which could more easily be considered in experiments. For $t<0$, we now consider an equilibrium situation for which the gas and liquid are in contact and for which the humidity of the gas is such that no evaporation takes place. The system is supposed at ambient pressure and temperature $\left(300^{\circ} \mathrm{K}, 1 \mathrm{~atm}\right)$, which corresponds for a waterPVA mixture to a saturation pressure $p_{\text {sat }}=3.52 \times 10^{-2} \mathrm{~atm}$. For a given water concentration in the liquid, Raoult's law allows then to determine the corresponding concentration in the gas. Then a sudden Joule expansion of the gas is allowed to take place at $t=0$, which keeps the temperature constant and decreases the (total) pressure $P_{t}$. Since the propagation of pressure perturbations is very fast, one can consider an immediate pressure decrease in the gas, which initiates evaporation, with a jump at the interface, similar to that described earlier. Fig. 7 is a plot of the concentration at the interface after the jump as a function of the postexpansion pressure Ptafter and for different initial concentrations in the liquid phase. As expected, the jump in the interfacial concentration increases with the imposed pressure drop and for sufficiently low post-expansion pressures and sufficiently low initial water concentrations in the mixture, immediate gelation can take place, preventing Marangoni convection. 


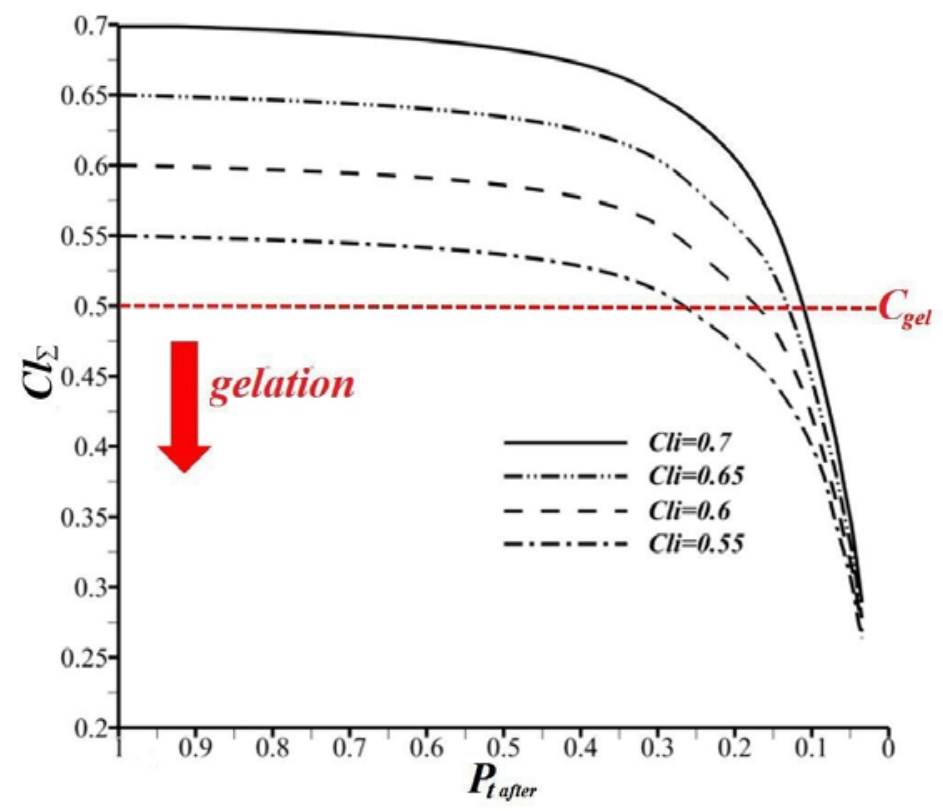

Fig. 7. Interfacial liquid mass fraction $\left(C l_{\Sigma}\right)$ after the jump as a function of the post-expansion pressure Ptafter (in atm).

\section{Conclusion}

In this paper, we have built a model for the drying process of a polymer solution taking into account the physics of the upper gas layer and its interactions with the liquid. During the drying process, the solvent evaporates, which results in a decrease of $C_{l}$ and of the thickness of the binary mixture. When the mass fraction of liquid reaches the so-called gelation mass fraction, a phase-change takes place near the free surface and the upper part of the mixture is not a liquid anymore. Then, as drying proceeds, the whole binary liquid layer tends towards gelation and a dryer and dryer gel layer progressively invades the whole system, until evaporation finally ceases. A large thickness of the gas layer leads to a slower drying process, because the evaporation rates is smaller in that situation. Our model has also been compared with previous approaches [20,22] for which a phenomenological law was introduced to describe the evaporation flux. In those works, the importance of the evaporation flux is described by a Peclet number and we proved that for appropriate values of this number, a good global agreement between our model and the other ones can be found. Otherwise stated, our model allows to relate the value of the purely empirical Peclet number to true physical quantities such as diffusion in the gas, condition at the top of the gas, etc., and thus to determine theoretically the value of the Peclet number corresponding to a given practical situation. Another important result of our work is the description of possible immediate gelation at the liquid-gas interface when evaporation is induced in a rather sudden way. This immediate gelation is related to the very high (theoretically infinite) evaporation flux predicted by our approach and impossible with the other models. This immediate gelation could thus prevent Marangoni convection in the system, which could clearly be of interested in some practical situations. 


\section{Acknowledgements}

We thank P. Colinet, B. Haut and B. Sobac (TIPs, University of Brussels) for interesting discussions. Financial support from F.R.S.-FNRS ("DITRASOL” PDR T.0123.16) and from BELSPO ("EVAPORATION” MAP-PRODEX project) is also acknowledged.

\section{Author contribution statement}

R.R. developed the theoretical formalism, performed the analytic calculations and the numerical simulations. P.C.D. and H.M. led the research work. All authors discussed the results and contributed to the final manuscript.

\section{Reference}

[1] P. L. Evans, L. W. Schwartz, R. V. Roy, J. Colloid Interface Sci. 227, 191(2000).

[2] S. D. Howison, J. A. Moriarty, J. R. Ockendon, E. L. Terrill, S. K. Wilson, J. Engrg. Math. 32, 377(1997).

[3] P. Mokarian-Tabari, M. Geoghegan, J. R. Howse, S. Y. Heriot, R. L. Thompson, R. A. L. Jones, Eur. Phys. J. E 33, 283(2010).

[4] S. Walheim, E. Schäffer, J. Mlynek, U. Steiner, Science 283, 520 (1999).

[5] A. Munch, C. P. Please, B. Wagner, Phys. Fluids 23, 102101 (2011).

[6] B. Guerrier, C. Bouchard, C. Allah, C. Benard, Aiche Journal 44, 791(1998).

[7] B. J. de Gans, U. S. Schubert, Langmuir 20, 7789 (2004).

[8] T. Kawase, T. Shimoda, C. Newsome, H. Sirringhaus, R. H.Friend, Thin Solid Films, 438-439, 279 ( 2003).

[9] B. J. de Gans, P.C. Duineveld, U.S. Schubert, Adv. Mater. 16, 203 (2004).

[10] B. Li, Y. P. Cao, X. Q. Feng, H. Gao, Soft Matter 8, 5728 (2012).

[11] A.F. Routh, Rep. Prog. Phys. 76, 046603 (2013).

[12] D. E. Bornside, C. W. Macosko, L. E. Scriven, J. Appl. Phys. 66, 5185(1989).

[13] P. G. de Gennes, Eur. Phys. J. E 7, 31 (2002). 
[14] M. Tsige and G. S. Grest, Macromolecules 37, 4333 (2004).

[15] Y. Reyes, Y. Duda, Langmuir 21, 7057 (2005).

[16] A.F. Routh, W.B. Zimmerman, Chem. Eng. Sci. 59, 2961 (2004).

[17] A.M. Konig, T. G. Weerakkody, J.L. Keddie, D. Johannsmann, Langmuir 24, 7580 (2008).

[18] A. Sarkar, M. S. Tirumkudulu, Langmuir 25, 4945 (2009).

[19] F. Buss, C.C. Roberts, K. S. Crawford, K. Peters, L. F. Francis, J. Colloid Interface Sci. 359, 112(2011).

[20] K.Y. Ozawa, T. Okuzono, M. DOI, Jpn. J. Appl. Phys. 45, 8817(2006).

[21] M. G. Hennessy, C. J. W. Breward, C. P. Please, Siam j. appl.math 76, 1711 (2016).

[22] M. G. Hennessy, G. L. Ferretti, J. T. Cabral, O. K. Matar, J. Colloid Interface Sci. 488, 61(2017).

[23] H. Machrafi, A. Rednikov, P. Colinet, P.C. Dauby, Eur. Phys. J. 192, 71(2011).

[24] T. Okuzono, M. Doi, Phys. Rev. E: Stat. Nonlinear Soft Matter Phys. 77, 1(2008).

[25] H. Machrafi, A. Rednikov, P. Colinet, P.C. Dauby, Phys. Rev. E 91, 053018 (2015).

[26] W. B. Russel, D. A. Saville, W. R. Schowalter: Colloidal Dispersions (Cambridge University Press, 1999).

[27] Y.S. Lipatov, A.E. Nesterov, Thermodynamics of Polymer Blends, Basel 1997.

[28] P. Atkins, Physical Chemistry: Chapter Simple Mixtures (Oxford University Press, Oxford, 2001).

[29] H. Machrafi, A. Rednikov, P. Colinet, P. C. Dauby, Phys. Fluids 25, 084106 (2013). 